\title{
Simultaneous Multi-Bragg Peak Coherent X-ray Diffraction Imaging
}

\author{
Florian Lauraux ${ }^{1}$, Stéphane Labat ${ }^{1}$, Sarah Yehya ${ }^{1}$, Marie-Ingrid Richard ${ }^{1,2}$, Steven J. Leake ${ }^{2}$, Tao Zhou ${ }^{2,3}$, \\ Jean-Sébastien Micha 4,5, Odile Robach 4,5, Oleg Kovalenko ${ }^{6}$, Eugen Rabkin ${ }^{6}$, Tobias U. Schülli ${ }^{2}$, \\ Olivier Thomas ${ }^{1}$ (D) and Thomas W. Cornelius ${ }^{1, *(D)}$
}

1 Aix Marseille Univ., Université de Toulon, CNRS, IM2NP, 13397 Marseille, France; florian.lauraux@im2np.fr (F.L.); stephane.labat@im2np.fr (S.L.); sarah.yehya@etu.univ-amu.fr (S.Y.); mrichard@esrf.fr (M.-I.R.); olivier.thomas@im2np.fr (O.T.)

2 ID01/ESRF-The European Synchrotron, 71 Avenue des Martyrs, 38000 Grenoble, France; steven.leake@esrf.fr (S.J.L.); nrszhou@gmail.com (T.Z.); schulli@esrf.fr (T.U.S.)

3 Nanoscience and Technology Division, Argonne National Laboratory, Lemont, IL 60439, USA

4 CRG-IF BM32 Beamline at the European Synchrotron (ESRF), CS40220, 38043 Grenoble CEDEX 9, France; micha@esrf.fr (J.-S.M.); robach@esrf.fr (O.R.)

5 Institut de recherche interdisciplinaire de Grenoble (CEA/IRIG), Université Grenoble Alpes, 17 rue des Martyrs, 38054 Grenoble, France

6 Department of Materials Science and Engineering, Technion-Israel Institute of Technology, Haifa 3200003, Israel; olegkov1@gmail.com (O.K.); erabkin@technion.ac.il (E.R.)

* Correspondence: thomas.cornelius@im2np.fr

Citation: Lauraux, F.; Labat, S.; Yehya, S.; Richard, M.-I.; Leake, S.J.; Zhou, T.; Micha, J.-S.; Robach, O.; Kovalenko, O.; Rabkin, E.; et al. Simultaneous Multi-Bragg Peak Coherent $X$-ray Diffraction Imaging. Crystals 2021, 11, 312. https:// doi.org/10.3390/cryst11030312

Academic Editor: Dina Carbone

Received: 15 February 2021

Accepted: 18 March 2021

Published: 22 March 2021

Publisher's Note: MDPI stays neutral with regard to jurisdictional claims in published maps and institutional affiliations.

Copyright: (c) 2021 by the authors. Licensee MDPI, Basel, Switzerland. This article is an open access article distributed under the terms and conditions of the Creative Commons Attribution (CC BY) license (https:// creativecommons.org/licenses/by/ $4.0 /)$

\begin{abstract}
The simultaneous measurement of two Bragg reflections by Bragg coherent X-ray diffraction is demonstrated on a twinned Au crystal, which was prepared by the solid-state dewetting of a $30 \mathrm{~nm}$ thin gold film on a sapphire substrate. The crystal was oriented on a goniometer so that two lattice planes fulfill the Bragg condition at the same time. The Au 111 and Au 200 Bragg peaks were measured simultaneously by scanning the energy of the incident $X$-ray beam and recording the diffraction patterns with two two-dimensional detectors. While the former Bragg reflection is not sensitive to the twin boundary, which is oriented parallel to the crystal-substrate interface, the latter reflection is only sensitive to one part of the crystal. The volume ratio between the two parts of the twinned crystal is about 1:9, which is also confirmed by Laue microdiffraction of the same crystal. The parallel measurement of multiple Bragg reflections is essential for future in situ and operando studies, which are so far limited to either a single Bragg reflection or several in series, to facilitate the precise monitoring of both the strain field and defects during the application of external stimuli.
\end{abstract}

Keywords: Bragg coherent X-ray diffraction imaging (BCDI); Au crystal; twin boundary

\section{Introduction}

In the last two decades, coherent X-ray diffraction imaging (CDI), which facilitates the three-dimensional mapping of nanostructured samples, has experienced tremendous progress. This lensless imaging technique retrieves the sample scattering function from coherent X-ray diffraction data using computational inversion algorithms to determine the phase of the scattered wave, which is not directly measured by a detector [1-3]. In the Bragg condition, the retrieved phase is directly related to the displacement field within the studied object. However, one single Bragg peak only provides access to the deformation along one single $q$-vector, thus severely limiting the information of the actual three-dimensional strain field of an object. In order to measure the deformation along several crystallographic directions and eventually obtain the complete strain tensor of a nanostructure, at least three independent Bragg peaks must be measured. The Bragg coherent diffraction imaging (BCDI) of six independent Bragg peaks on a single defect-free $\mathrm{ZnO}$ nanorod was demonstrated, rendering a spatial resolution of $40 \mathrm{~nm}$ [4]. The reconstruction of GaN nanowires 
containing inversion domain boundaries was demonstrated recently by measuring five independent Bragg peaks of the same nanorod, rendering a precision of $1 \mathrm{pm}$ in the displacement field and a spatial resolution of $10 \mathrm{~nm}$ [5]. In addition, Hofmann et al. reported 3D lattice distortions and the defect structure in ion-implanted nano-crystals inferred from the measurement of three independent Bragg reflections [6]. While the measurement of several Bragg peaks does not pose major problems for ex-situ BCDI experiments, in situ BCDI experiments are so far restricted to single Bragg reflections and, thus, are strongly limited regarding the displacement field and the detection and identification of defects due to the $G \cdot b$ criterion, i.e., that dislocations with the Burger's vector $b$ being perpendicular to the scattering vector $G(G \cdot b=0)$ are actually invisible.

In the case of reversible deformations, it is possible to measure several Bragg peaks consecutively in consecutive cycles of external stimuli, as demonstrated during hydrogen peroxide decomposition on Pt nanoparticles [7]. However, for imaging irreversible changes such as plasticity and the nucleation and evolution of defects and dislocation networks, consecutive measurements image different deformation states of the structure under study. Therefore, the standard BCDI approach of measuring a single Bragg reflection at a time is not able to give a full picture of the strain and defect evolution in functional materials.

In the present work, we present the simultaneous measurement of two independent Bragg peaks on a twinned Au crystal by multi-Bragg coherent X-ray diffraction imaging using two detectors. The nanostructure is oriented using the HKL mode of the synchrotron beamline operation software SPEC [8] so that two independent lattice planes are in the Bragg condition for the same incident angle and the same incident $X$-ray wavelength. In this manner, the symmetric Au 111 Bragg peak that is not sensitive to the twin boundary and the Au 200 Bragg reflection that is sensitive only to one twin grain are measured at the same time. Instead of rocking the sample, the energy of the incident X-ray beam is scanned to avoid any vibrations, as recently demonstrated by Cha et al. [9] and Lauraux et al. [10]. This multi-wavelength multi-Bragg peak approach allows for future in situ and operando experiments on functional materials exposed to external stimuli.

\section{Materials and Methods}

Nominally defect-free Au crystals were prepared on a $c$-oriented sapphire substrate by the solid-state dewetting of a gold thin film with an initial thickness of $30 \mathrm{~nm}$. Prior to annealing, this thin film was lithographically patterned with $2 \mu \mathrm{m}$ holes and a $50 \mu \mathrm{m}$ pitch, eventually resulting in individual gold crystals with lateral sizes of $\sim 500 \mathrm{~nm}$ and a height of $\sim 300 \mathrm{~nm}$, which are located in the center of a square with a side length of $50 \mu \mathrm{m}$. About one third of these gold crystals contain a twin boundary parallel to the crystal-substrate interface, as inferred from Laue microdiffraction.

Laue microdiffraction was performed at the BM32 beamline at the European Synchrotron Radiation Facility (ESRF) in Grenoble (France). The incident polychromatic X-ray beam with an energy bandwidth of $5-25 \mathrm{keV}$ was focused down to $500 \mathrm{~nm}(\mathrm{H}) \times 500 \mathrm{~nm}(\mathrm{~V})$ on the sample surface using a pair of Kirkpatrick-Baez (KB) mirrors. The sample surface was inclined by $40^{\circ}$ with respect to the incident $X$-ray beam and the diffracted $X$-rays were recorded by a PhotonicScience sCMOS detector with a pixel size of $70 \mu \mathrm{m} \times 70 \mu \mathrm{m}$, which was installed at $90^{\circ}$ in a (calibrated) distance of $77 \mathrm{~mm}$ from the sample position. The Au crystals were located by two-dimensional X-ray fluorescence (XRF) mapping of the Au L $\mathrm{L}_{\mathrm{III}}$ fluorescence yield using a Ketek SDD energy-resolved point detector. Laue microdiffraction patterns were recorded at the position with the maximum $\mathrm{Au} \mathrm{L}_{\mathrm{III}}$ fluorescence yield and were indexed using the LaueTools software [11]. A background microdiffraction pattern was recorded at a distance of few micrometers from the Au crystal on the bare substrate and then subtracted from the Laue microdiffraction pattern obtained at the position of the crystal, thus removing the sapphire substrate Laue spots and improving the visibility of the Au Laue peaks.

The Bragg coherent $X$-ray diffraction experiment was performed at the ID01 beamline at the ESRF. The incident $9 \mathrm{keV} X$-ray beam was focused using a pair of $\mathrm{KB}$ mirrors. 
The coherent part of the incident X-ray beam was selected by closing slits located in front of the KB mirrors to a size of $60 \mu \mathrm{m}(\mathrm{H}) \times 200 \mu \mathrm{m}(\mathrm{V})$. The focal spot size of this coherent X-ray beam amounted to $175 \mathrm{~nm}(\mathrm{~V}) \times 360 \mathrm{~nm}(\mathrm{H})$. By closing the coherence slits further to $50 \mu \mathrm{m}(\mathrm{H}) \times 30 \mu \mathrm{m}(\mathrm{V})$, thus reducing the numerical aperture of the focusing optics, the focal spot size was increased to $590 \mathrm{~nm}(\mathrm{H}) \times 580 \mathrm{~nm}(\mathrm{~V})$, matching the lateral dimensions of the Au crystals. The focal profile of the beam was characterized using a $2 \mathrm{D}$ ptychography [12] approach on a test pattern featuring a $40 \mu \mathrm{m}$-diameter tungsten Siemens star placed close to the focal position of the KB mirrors [13]. The tungsten structure and the complex-valued wavefront were retrieved simultaneously using the ptychography reconstruction code of the PyNX package [14].

Employing the HKL mode of the SPEC-software used for operating the high-precision diffractometer at the ID01 beamline, the twinned Au crystals are oriented so that the $\mathrm{Au}$ 111, as well as the Au 200, lattice plane fulfills the Bragg condition at the same angle of incidence of $18.32^{\circ}$ and at the same energy of $9 \mathrm{keV}$ of the incident X-ray beam. The Au 111 Bragg reflection was recorded by an Eiger $2 \mathrm{M}$ detector with a pixel size of $75 \mu \mathrm{m} \times 75 \mu \mathrm{m}$ mounted on the diffractometer arm at a distance of $1.32 \mathrm{~m}$ from the sample position, while the Au 200 Bragg reflection was monitored by a MAXIPIX detector with a pixel size of $55 \mu \mathrm{m} \times 55 \mu \mathrm{m}$ installed on a separate mounting at a distance of $1.37 \mathrm{~m}$. The experimental setup is illustrated in the photo and the schematics in Figure 1a-c. As indicated in the schematic side and top views of the experimental setup, the reciprocal space $q_{\mathrm{x}}$ direction is

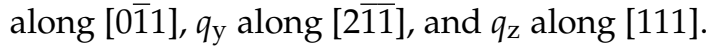

Three-dimensional reciprocal space maps of the Au 111 and Au 200 Bragg peaks were recorded simultaneously by scanning the energy of the incident X-ray beam by $+/-250 \mathrm{eV}$ in steps of $2 \mathrm{eV}[10,15]$. To keep the incident intensity constant, the undulator gaps were adjusted every 5 energy steps (corresponding to $10 \mathrm{eV}$ ). The fact that the diffraction signal is probed along $q_{\mathrm{z}}$ during energy scans limits the information within the $q_{\mathrm{x}}-q_{\mathrm{y}^{-}}$ plane. Therefore, the $2 \theta$ angle of the detector monitoring the Au 111 Bragg peak was varied simultaneously by $-/+1.0^{\circ}$ in steps of 8 millidegrees, thus eventually probing the reciprocal space in the same manner as during rocking scans.

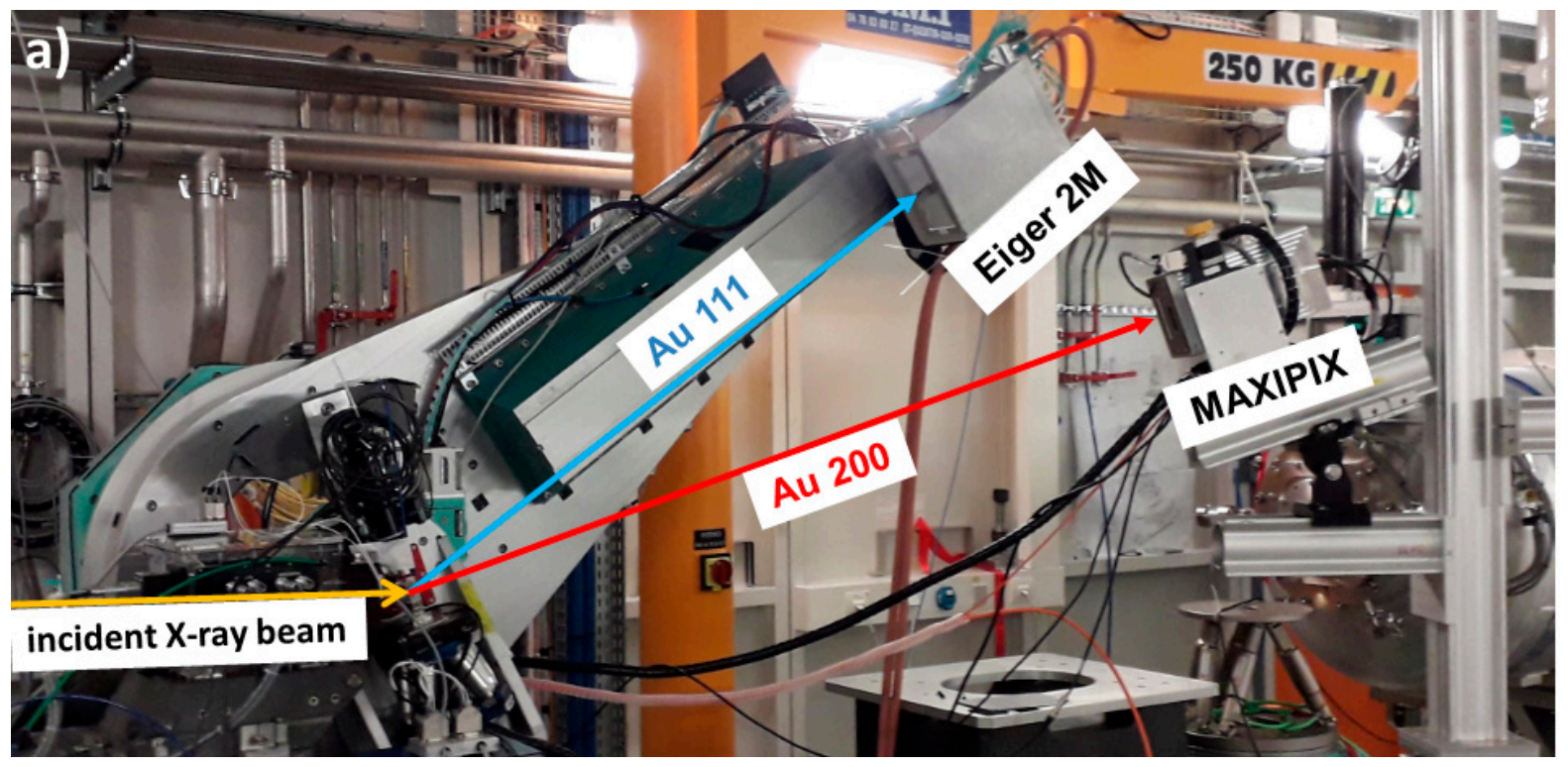

Figure 1. Cont. 


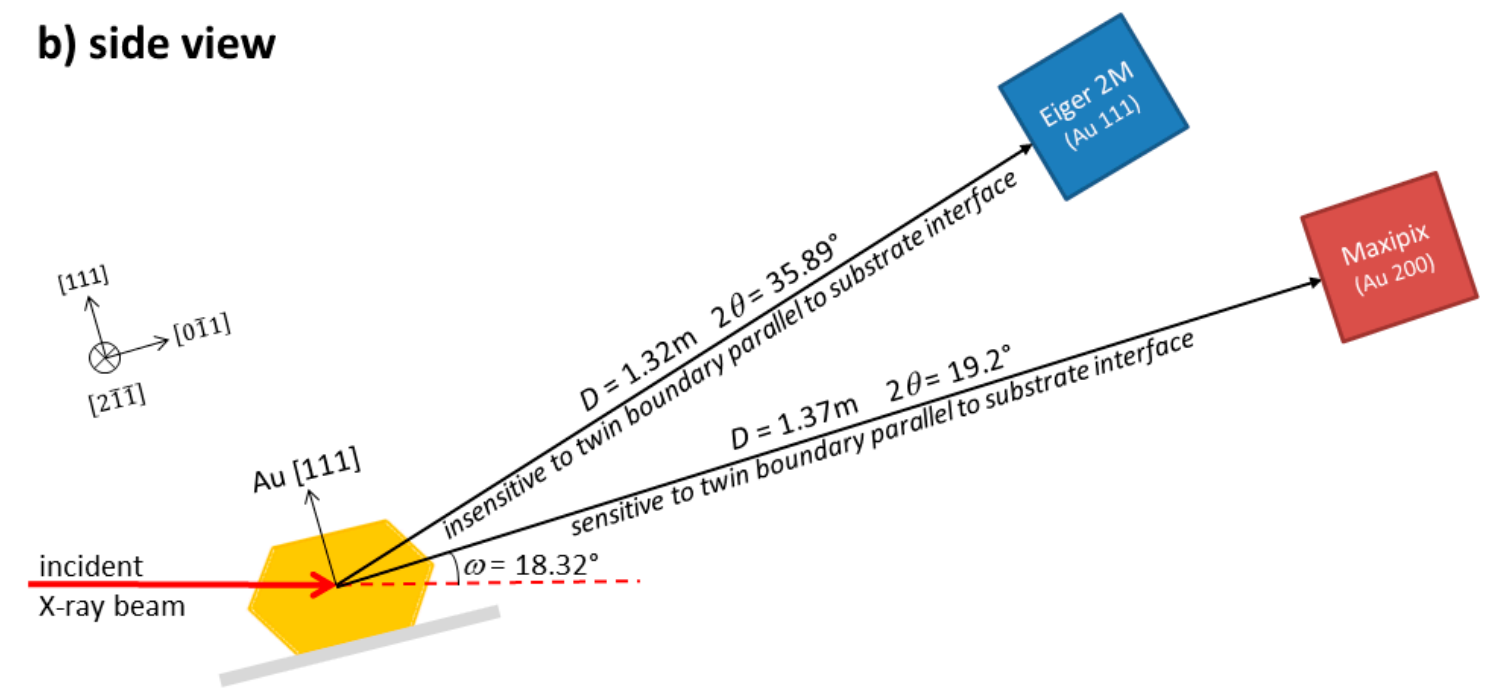

\section{c) top view}

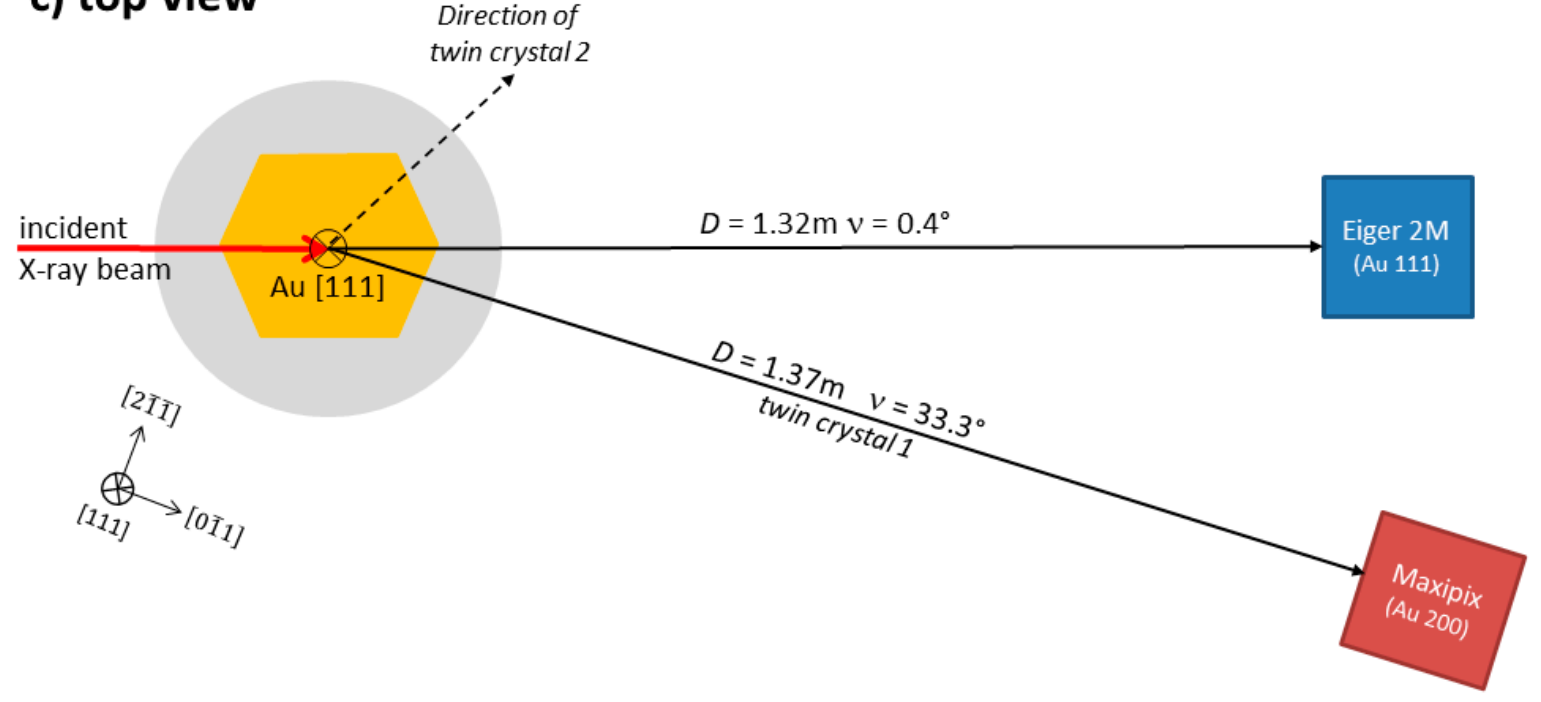

Figure 1. (a) Photograph, (b) schematic side view, and (c) schematic top view of the experimental setup at the ID01 beamline at ESRF using two 2D detectors simultaneously. $\omega, \gamma$, and $2 \theta$ correspond to the incident angle (similar for the two reflections), and the in-plane and out-of-plane scattering angles.

\section{Results}

A scanning XRF map of the Au $\mathrm{L}_{\mathrm{III}}$ fluorescence yield is presented in the inset of Figure 2, showing a part of the square pattern with a single Au crystal in its center (which is located here at the right edge of the XRF map) highlighted by a circle. At the position of the Au crystal, a Laue microdiffraction pattern was recorded. Laue microdiffraction patterns recorded at the Au crystal position and a few micrometers away on the bare substrate are presented in Figure S1 in the Supplementary Material. The background-subtracted Laue microdiffraction pattern for this Au crystal is shown in Figure 2. The indexation process shows the presence of a twin boundary parallel to the crystal-substrate interface, i.e., parallel to (111). As demonstrated by the indexed pattern shown in Figure S2 in the Supplementary Material, the diffraction spots can be fitted well assuming a $\sum 3$ relation between the two parts of the crystal (here, $\sum$ is a reciprocal density of coincident sites of the two misoriented lattices). While the central Au 222 Laue spot is identical for both crystal parts, the zone axes are rotated by $180^{\circ}$ with respect to each other. The $180^{\circ}$ rotation of the zone axes for the two crystal parts comprises the angle of $120^{\circ}$ between two zone axes for a face-centered cubic crystal and the $\sum 3$ relation between the two parts of the crystal inducing an additional rotation of $60^{\circ}$. As the lattice planes are rotated by $60^{\circ}$ and, thus, 
diffract to different $\chi$ (azimuthal) and $2 \theta$ (scattering) angles, the energy of the diffracted $\mathrm{X}$-rays is different in order to fulfil the Bragg condition.

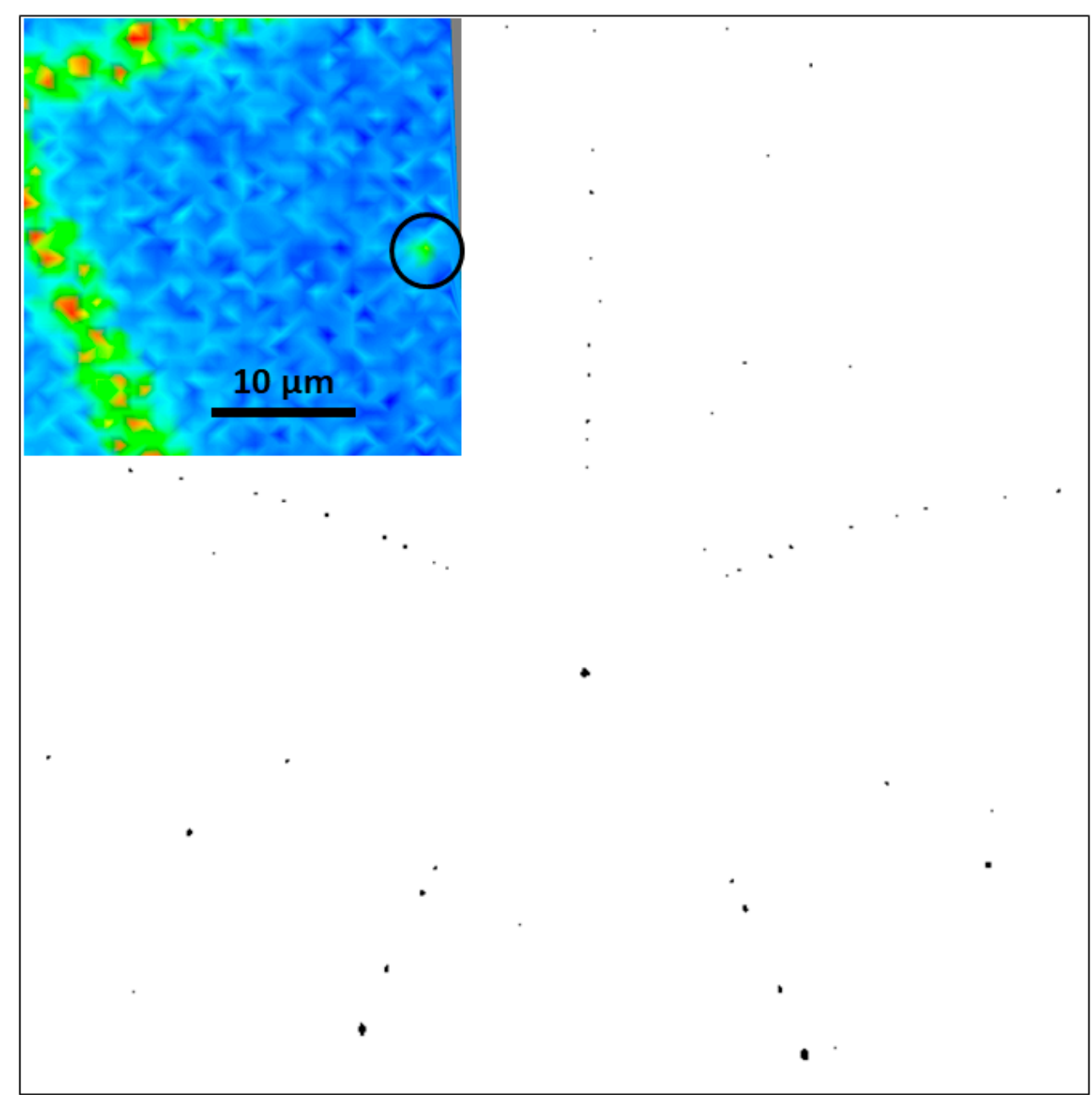

Figure 2. Background-subtracted Laue microdiffraction pattern of an Au crystal. The inset shows an X-ray fluorescence map of the Au $\mathrm{L}_{\mathrm{III}}$ fluorescence yield. The selected Au crystal is marked by a circle.

The intensity profile of the diffraction peaks as a function of the energy of the diffracted X-rays can be fitted well by a polynomial function (as shown in Figure S3 in the Supplementary Material). The integrated intensity of the intensity-energy profile provides access to the volume of the diffracting crystal. Thus, comparing the integrated intensity for the two sub-crystals of a twinned crystal allows the determination of the volume ratio of the two sub-crystals. However, it should be noted that for small Miller indices, multiple energies of the diffracted X-rays are possible due to the fact that the diffraction peaks of the same family (e.g., $\{111\}$ lattice planes) are located on the same position on the detector for Laue diffraction. Therefore, these diffraction spots are discarded in the calculation of the volume ratio of the two sub-crystals. The volume ratio for the twinned Au crystal presented in Figure 2 amounts to 1:11.

Figure 3 presents the sequences of two-dimensional coherent $\mathrm{X}$-ray diffraction patterns that were recorded in the vicinity of the $\mathrm{Au} 111$ and $\mathrm{Au} 200$ Bragg peaks using an Eiger 2M and a MAXIPIX detector, respectively, while scanning the energy of the incident $X$-ray beam. This scan was performed from smaller toward higher X-ray energies, thus scanning the detectors in reciprocal space from smaller to larger $q$-values. Both detectors show slices of the facet streaks of the Au crystal at the beginning and toward the end of the measurement. However, they do not cut through the center of the respective Bragg reflection at the 
same energy but with an offset of $<30 \mathrm{eV}$. This discrepancy in alignment is not of major importance as long as the scan range in energy is sufficiently large to cover the complete three-dimensional reciprocal space information of the Bragg peaks.

\begin{tabular}{|c|c|c|c|c|c|c|c|}
\hline $8942 \mathrm{eV}$ & $8972 \mathrm{eV}$ & $9002 \mathrm{eV}$ & $9032 \mathrm{eV}$ & $9062 \mathrm{eV}$ & $9092 \mathrm{eV}$ & $9122 \mathrm{eV}$ & $9152 \mathrm{eV}$ \\
\hline Au 111 & Hot & & & & & & \\
\hline
\end{tabular}

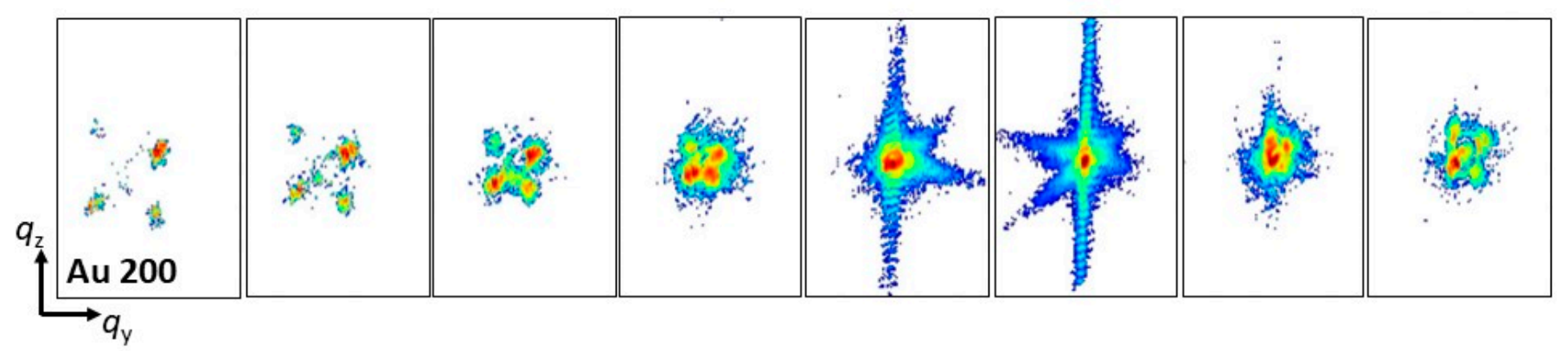

Figure 3. Sequences of 2D coherent diffraction patterns of the Au 111 and Au 200 Bragg peaks during a scan of the energy of the incident $\mathrm{X}$-ray beam.

The three-dimensional reciprocal space maps of the Au 111 and Au 200 Bragg peaks are shown in Figure 4a. Both three-dimensional Bragg reflections have the same shape with the same streaks originating from the side facets. The Bragg electron density and the phase were reconstructed from the 3D Bragg coherent diffraction patterns (BCDPs) using the PyNX software. The multi-wavelength BCDI data were pre-interpolated onto a matrix of uniformly spaced $q_{\mathrm{x}}, q_{\mathrm{y}}, q_{\mathrm{z}}$, before the phase retrieval process. The effect of pre- and post-interpolation of the raw data can be found in [10]. An isosurface representation of the three-dimensional shapes of the crystals reconstructed from the Au 111 and Au 200 Bragg peaks are presented in Figure $4 \mathrm{~b}$. Both crystals have similar shapes. However, the thickness of the crystal reconstructed from the Au 111 Bragg peak is $370 \mathrm{~nm}$, whereas the crystal reconstructed from the Au 200 Bragg reflection measures only $330 \mathrm{~nm}$. While the Au 111 Bragg peak is not sensitive to the twin boundary parallel to the substrate interface, and thus represents the diffraction signal of the entire crystal, the Au 200 reflection corresponds to the upper part of the twinned crystal, thus explaining the difference in the thickness. Therefore, the second part of the twinned crystal measures $40 \mathrm{~nm}$ in height. These thicknesses are in very good agreement with reconstructions obtained from classical rocking scans performed separately at each reflection, being sensitive to the complete crystal and to one of the two variants: $\mathrm{Au}$ 111, Au 200, and Au 002 (as demonstrated below). Hence, the thickness proportion of the two parts of the twinned crystal amounts to 1:9. This ratio is comparable to the volume ratio determined by Laue microdiffraction. As the crystals are not cubic but have a Winterbottom shape with inclined facets, the volume ratio differs from the thickness proportion of the two crystal parts.

The vertical cross-sections through the reconstructed crystals are shown in Figure 4c displaying the displacement along the respective $q$-vector. The displacement field is different for the $\mathrm{Au} 111$ and for the $\mathrm{Au} 200$, indicating that the displacement field fluctuation is larger for the component along the 111 direction.

For comparison, Bragg coherent X-ray diffraction images of the Au 111, Au 200, as well as Au 002 (corresponding to the second variant of the twinned Au crystal) reflections were recorded individually by ordinary rocking scans using solely the MAXIPIX detector installed on the detector arm of the diffractometer. Here, the crystal was rotated by $60^{\circ}$ around $\phi$ in order to align either the upper or the lower variant in the Bragg condition. The iso-surfaces of the three-dimensional reciprocal space maps of the three abovementioned 
Bragg reflections are presented in Figure 5. The size fringes of the vertical CTR for the upper part of the twinned crystal (Au 200) are similar to those of the entire crystal (Au 111), while the size fringes for the second part of the crystal have a significantly lower periodicity, indicating a significantly smaller thickness of this second part of the crystal compared to the first one. The directions of the other streaks, as well as the distance between the size fringes, are the same for the larger part of the crystal and the crystal in its whole. The shapes of these crystals are thus very similar, showing the same side facets and having the same lateral extension. For the smaller crystal part, however, the streaks from the side facets of the crystal are not visible, probably due to the very small diffractive volume of this variant.

(a)

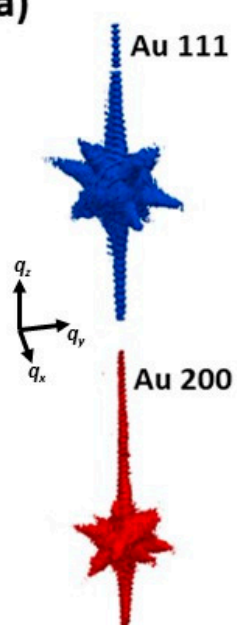

(b)

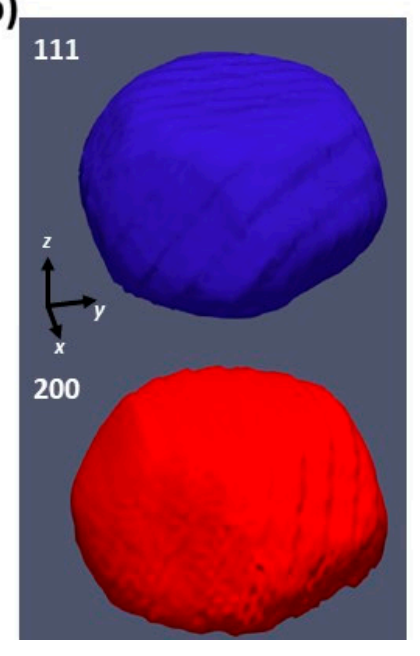

(c)

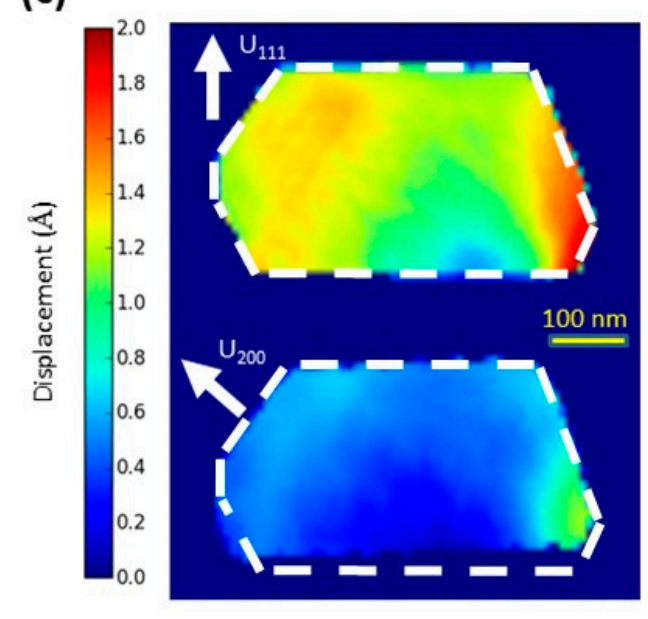

Figure 4. (a) 3D reciprocal space maps of the Au 111 and Au 200 Bragg peaks measured simultaneously using an Eiger 2 M and a MAXIPIX detector. (b) Isosurface of the Bragg electronic density reconstructed object from the Au 111 and 200 Bragg peaks. (c) Vertical cuts of the 3D displacement field component. The dotted white contours are guides for the eyes and delimit the shape of the crystal measured at the Au 111 Bragg reflection.
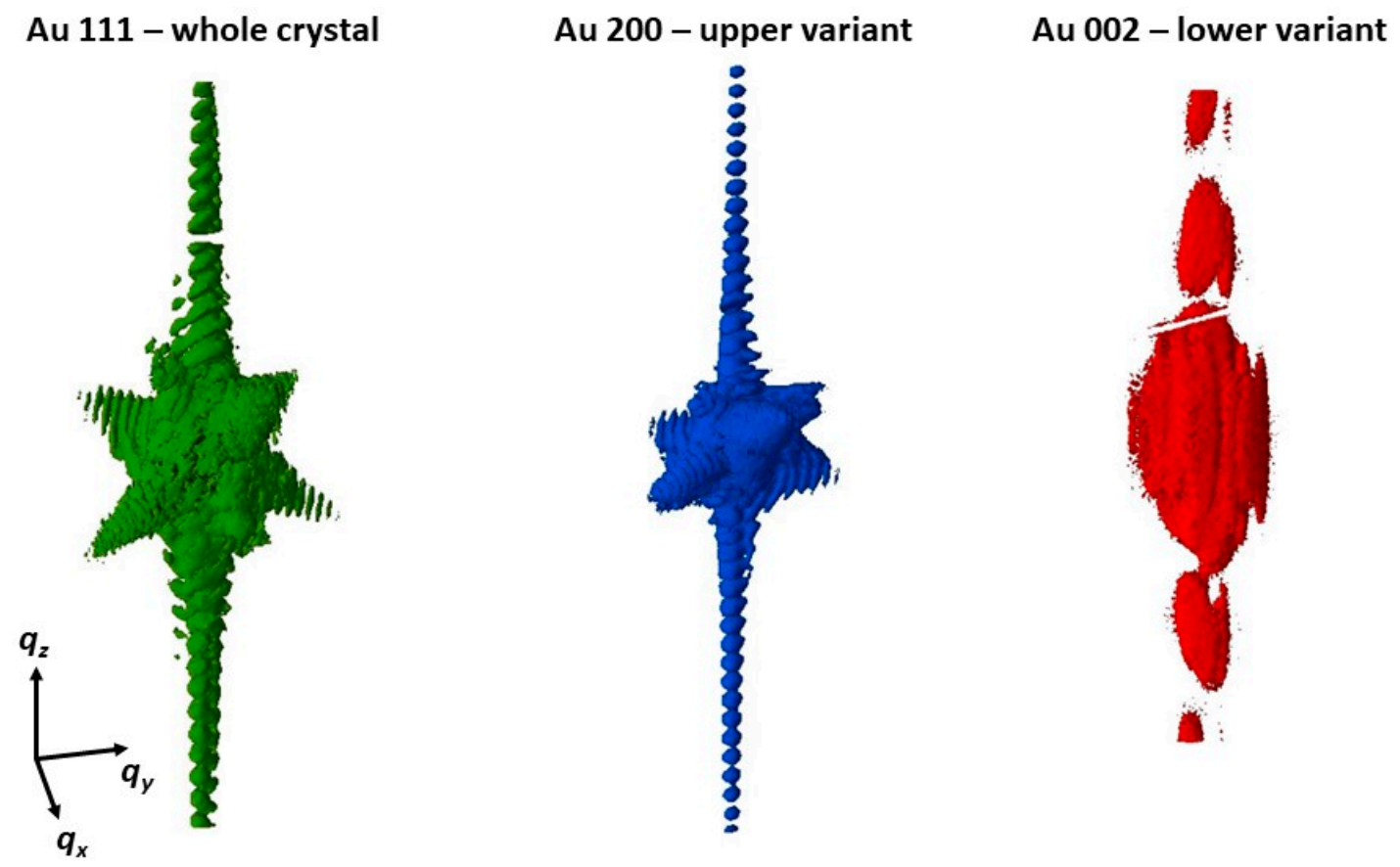

Figure 5. Bragg coherent X-ray diffraction patterns of the Au 111, Au 200, and Au 002 Bragg reflections for a twinned Au crystal where the twin boundary is parallel to the crystal-substrate interface. 
The Bragg electron density displayed in Figure 6 was reconstructed from the Bragg coherent X-ray diffraction patterns presented in Figure 5 using the PyNX code. The two parts of the crystal have thicknesses of 330 and $40 \mathrm{~nm}$, which agrees very well with the total thickness of the entire reconstructed crystal of $370 \mathrm{~nm}$. The thicknesses for the whole crystal and the upper variant are in excellent agreement with the results obtained by the multiwavelength multi-Bragg peak CDI presented above.
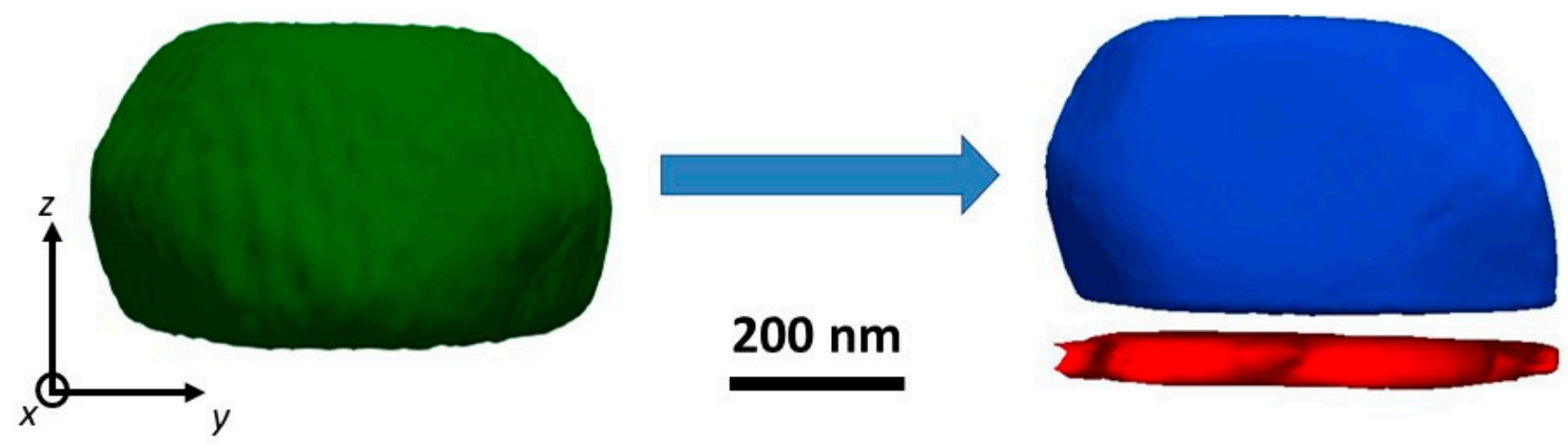

\section{$200 \mathrm{~nm}$}

Figure 6. Bragg electron density of the twinned Au crystal reconstructed from the 3D Bragg coherent X-ray diffraction patterns shown in Figure 5. The left-hand side shows the reconstruction of the entire crystal reconstructed from the Au 111 Bragg reflection, while the right-hand side shows the two variants reconstructed from the Au 200 and Au 002 Bragg reflections.

\section{Discussion}

Here, the voxel sizes of the reconstructions from the multiwavelength multi-Bragg peak CDI amount to $14 \times 11 \times 15$ and $55 \times 13 \times 13 \mathrm{~nm}^{3}$ for the $\mathrm{Au} 111$ and $\mathrm{Au} 200$ reflections, respectively. The increased voxel size in one direction for the Au $200 \mathrm{Bragg}$ reflection originates from the fact that the volume probed in reciprocal space is smaller along $q_{\mathrm{x}}$, a coordinate of the scattering vector along the beam direction. This limitation is due to the rigid and fixed mounting of the second detector. Larger distances in reciprocal space can be probed when installing the detector on motorized stages.

The reconstructions of the entire crystal and the upper variant obtained from multiwavelength multi-reflection BCDI were found to be in excellent agreement with reconstructions obtained from separate rocking scans of the three independent Bragg peaks $\mathrm{Au}$ 111, Au 200, and Au 002, employing a single detector. The HKL mode of the operation software allows for the alignment of the crystal with three independent lattice planes fulfilling the Bragg condition, so that three Bragg peaks could be monitored simultaneously when installing three detectors. This would eventually allow for the probing of the entire crystal and the two variants of the twinned crystal. Here, the proof-of-concept was demonstrated on two independent Bragg reflections, enabling the recovery of two independent components of the displacement vector in one of the two variants of the twinned crystal.

The volume ratio for the twinned Au particle determined in the present work indicates that the distance of the twin boundary from the substrate corresponds approximately to the initial film thickness prior to dewetting $(30 \mathrm{~nm})$. In this respect, it is worth mentioning that Amram et al. observed the formation of twin boundaries parallel to the substrate, and at the distance from the substrate corresponding to the original film thickness, during the intermediate stages of the solid-state dewetting of $\mathrm{Au}(\mathrm{Fe})$ thin films deposited on sapphire [16]. It was proposed that while the energy penalty associated with such twin boundaries is relatively low, they may strongly facilitate dewetting kinetics by reducing the energy barrier for shape-changing surface diffusion.

In addition, the fact that about one-third of the studied particles contained a twin boundary may explain the high scatter of the compressive strength of such particles observed in several experimental studies [17,18]. Indeed, both the experimental data [19] and atomistic computer simulations [20] indicate that twin boundaries in otherwise defect- 
free metallic nanostructures assist in the heterogeneous nucleation of dislocations and reduce the mechanical strength.

\section{Conclusions}

In conclusion, the simultaneous Bragg coherent X-ray diffraction imaging of two Bragg reflections was applied to a twinned Au crystal. While one of the Bragg peaks, the $\mathrm{Au} 111$, was not sensitive to the twin boundary oriented parallel to the crystal-substrate interface, the other Bragg reflection (Au 200) was only sensitive to one of the two variants that represented the top part of the crystal. The reconstructed Bragg electron density is in very good agreement with reconstructions obtained from classical rocking scans performed at each of the Bragg reflections independently. This multiple-Bragg peak BCDI technique brings significant advantages for in situ and operando experiments probing the strain field in different $q$ directions simultaneously while the sample is exposed to external stimuli. By contrast, the consecutive measurement of single Bragg peaks, as it has been done until now, may probe different crystal states in the case of irreversible phenomena like plasticity, fatigue, or fracture. The presented multi-Bragg peak BCDI can be further improved by mounting the detectors on motorized stages, allowing the probing of a larger volume in reciprocal space and, thus, improving the real-space resolution. In addition, the installation of three detectors and the simultaneous measurement of three independent Bragg reflections like the Au 220 situated at about $42^{\circ}$ in $2 \theta$ for an incident angle of $18.32^{\circ}$ at $9 \mathrm{keV}$ as in the present work would eventually provide access to the full strain tensor.

Supplementary Materials: The following are available online at https:/ / www.mdpi.com/2073-4 352/11/3/312/s1, Figure S1: Laue microdiffraction pattern (a) of the twinned gold crystal on a sapphire substrate, (b) a few micrometers away from the Au crystal on the bare substrate, and (c) after subtraction of the substrate background pattern. Figure S2: Indexed Laue microdiffraction patterns of one Au crystal assuming that the two parts of the crystal have a 3 relation. Figure S3: Intensity spectrum of the incident polychromatic X-ray beam superimposed with the intensity of the indexed Laue spots for the two variants of the twinned gold crystal and the energy of the respective diffracted X-rays.

Author Contributions: Conceptualization, T.W.C., S.L. and O.T.; methodology, S.J.L., T.Z., J.-S.M., O.R. and T.U.S.; formal analysis, F.L., S.Y. and S.L.; investigation, F.L., S.L., M.-I.R., O.K., O.T. and T.W.C.; writing—original draft preparation, T.W.C.; writing—review and editing, T.W.C., S.L., M.-I.R., S.J.L., E.R. and O.T.; supervision, T.W.C. and S.L.; funding acquisition, E.R. and M.-I.R. All authors have read and agreed to the published version of the manuscript.

Funding: This research was partially funded by a grant from the Ministry of Science and Technology, Israel and France's Centre National de la Recherche Scientifique (CNRS). This project has further received funding from the European Research Council (ERC) under the European Union's Horizon 2020 research and innovation program (grant agreement No. 818823).

Acknowledgments: The authors gratefully acknowledge both the European Synchrotron Radiation Facility and the French CRG program committee for beamtime allocation at the ID01 beamline and the BM32 beamline. They further thank the beamline staff for excellent support during the measurement campaigns. The thin film processing was performed at the Micro-Nano Fabrication and PrintingUnit (MNF and PU), Technion. This research was partially supported by a grant from the Ministry of Science and Technology, Israel and France's Centre National de la Recherche Scientifique (CNRS).

Conflicts of Interest: The authors declare no conflict of interest.

\section{References}

1. Fienup, J.R. Reconstruction of an object from the modulus of its Fourier transform. Opt. Lett. 1978, 3, 27-29. [CrossRef] [PubMed]

2. Fienup, J.R. Phase retrieval algorithms: A comparison. Appl. Opt. 1982, 21, 2758-2769. [CrossRef] [PubMed]

3. Marchesini, S.; He, H.; Chapman, H.N.; Hau-Riege, S.P.; Noy, A.; Howells, M.R.; Weierstall, U.; Spence, J.C.H. X-ray image reconstruction from a diffraction pattern alone. Phys. Rev. B 2003, 68, 140101. [CrossRef]

4. Newton, M.C.; Leake, S.J.; Harder, R.; Robinson, I.K. Three-dimensional imaging of strain in a single ZnO nanorod. Nat. Mater. 2010, 9, 120-124. [CrossRef] [PubMed] 
5. $\quad$ Labat, S.; Richard, M.-I.; Dupraz, M.; Gailhanou, M.; Beutier, G.; Verdier, M.; Mastropietro, F.; Cornelius, T.W.; Schülli, T.U.; Eymery, J.; et al. Inversion domain boundaries in GaN nanowires revealed by coherent Bragg imaging. ACS Nano 2015, 9, 9210-9216. [CrossRef] [PubMed]

6. Hofmann, F.; Tarleton, E.; Harder, R.J.; Philips, N.W.; Ma, P.-W.; Clark, J.N.; Abbey, B.; Liu, W.; Beck, C.E. 3D lattice distortions and defect structures in ion-implanted nano-crystals. Sci. Rep. 2017, 7, 45993. [CrossRef] [PubMed]

7. Choi, S.; Chung, M.; Kim, D.; Kim, S.; Yun, K.; Cha, W.; Harder, R.; Kawaguchi, T.; Liu, Y.; Ulvestad, A.; et al. In situ strain evolution on Pt nanoparticles during hydrogen peroxide decomposition. Nano Lett. 2020, 20, 8541-8548. [CrossRef] [PubMed]

8. Certified Scientific Software. Available online: https:/ / certif.com/ (accessed on 20 March 2021).

9. Cha, W.; Ulvestad, A.; Allain, M.; Chamard, V.; Harder, R.; Leake, S.J.; Maser, J.; Fuoss, P.H.; Hruszekewycz, S.O. Threedimensional variable-wavelength X-ray Bragg coherent diffraction imaging. Phys. Rev. Lett. 2016, 117, 225501. [CrossRef] [PubMed]

10. Lauraux, F.; Cornelius, T.W.; Labat, S.; Richard, M.-I.; Leake, S.; Zhou, T.; Kovalenko, O.; Rabkin, E.; Schülli, T.U.; Thomas, O. Multi-wavelength Bragg coherent X-ray diffraction imaging of Au particles. J. Appl. Cryst. 2020, 53, 170-177. [CrossRef]

11. Micha, J.-S. LaueTools: Open Source Python Packages for X-ray MicroLaue Diffraction Analysis. 2014. Available online: https:/ / gitlab.esrf.fr/micha/lauetools (accessed on 20 March 2021).

12. Pfeiffer, F. X-ray ptychography. Nat. Photonics 2018, 12, 9-17. [CrossRef]

13. Leake, S.J.; Favre-Nicolin, V.; Zatterin, E.; Richard, M.-I.; Fernandez, S.; Chahine, G.; Zhou, T.; Boesecke, P.; Djazouli, H.; Schülli, T.U. Coherent nanoscale X-ray probe for crystal interrogation at ID01, ESRF-The European Synchrotron. Mater. Des. 2017, 119, 470-471. [CrossRef]

14. Mandula, O.; Aizarna, M.E.; Eymery, J.; Burghammer, M.; Favre-Nicolin, V. PyNX.Ptycho: A computing library for X-ray coherent diffraction imaging of nanostructures. J. Appl. Cryst. 2016, 49, 1842. [CrossRef]

15. Cornelius, T.W.; Davydok, A.; Jacques, V.L.R.; Grifone, R.; Schülli, T.; Richard, M.-I.; Beutier, G.; Verdier, M.; Metzger, T.H.; Pietsch, U.; et al. In situ 3D reciprocal space mapping during mechanical deformation. J. Synchrotron Radiat. 2012, 19, 688-694. [CrossRef] [PubMed]

16. Amram, D.; Klinger, L.; Rabkin, E. Anisotropic hole growth during solid-state dewetting of single crystal Au-Fe thin films on sapphire. Acta Mater. 2012, 60, 3047-3056. [CrossRef]

17. Mordehai, D.; Lee, S.-W.; Backes, B.; Srolovitz, D.J.; Nix, W.D.; Rabkin, E. Size effect in compression of single-crystal gold microparticles. Acta Mater. 2011, 59, 5202-5215. [CrossRef]

18. Flanagan, T.J.; Kovalenko, O.; Rabkin, E.; Lee, S.-W. The effect of defects on strength of gold microparticles. Scr. Mater. 2019, 171, 83-86. [CrossRef]

19. Casillas, G.; Palomares-Báez, J.P.; Rodríguez-López, J.L.; Luo, J.; Ponce, A.; Esparza, R.; Velázquez-Salazar, J.J.; Hurtado-Macias, A.; González-Hernández, J.; José-Yacaman, M. In situ TEM study of mechanical behaviour of twinned nanoparticles. Philos. Mag. 2012, 92, 4437-4453. [CrossRef]

20. Hyde, B.; Espinosa, H.D.; Farkas, D. An atomistic investigation of elastic and plastic properties of Au nanowires. JOM 2005, 57, 62-66. [CrossRef] 